\title{
Noninvasive bunch length measurements exploiting Cherenkov diffraction radiation
}

A. Curcio, M. Bergamaschi, R. Corsini, W. Farabolini, D. Gamba, L. Garolfi, R. Kieffer, T. Lefevre, and S. Mazzoni CERN, CH-1211, Geneva, Switzerland

K. Fedorov

John Adams Institute at Royal Holloway, University of London, Egham, Surrey, TW20 OEX, United Kingdom

and Tomsk Polytechnic University, 634050, Lenin Ave, Tomsk, Russia

J. Gardelle

CEA, F-33114 Le Barp, France

A. Gilardi

CERN, CH-1211, Geneva, Switzerland and University of Naples Federico II, Naples, Italy

P. Karataev

John Adams Institute at Royal Holloway, University of London, Egham, Surrey, TW20 OEX, United Kingdom

K. Lekomtsev

National Research Nuclear University MEPhI (Moscow Engineering Physics Institute),

Kashirskoe Highway, 31, Moscow 115409, Russian Federation and John Adams Institute at Royal Holloway, University of London, Egham, Surrey, TW20 OEX, United Kingdom

T. Pacey and Y. Saveliev

ASTeC, STFC Daresbury Labaratory, WA4 4AD, United Kingdom and Cockcroft Institute, Daresbury, Warrington, WA4 4AD, United Kingdom

A. Potylitsyn

Tomsk Polytechnic University, 634050, Lenin Ave, Tomsk, Russia

E. Senes

John Adams Institute and University of Oxford, The Denys Wilkinson Building, Keble Road, Oxford OXI 3RH, United Kingdom and CERN, CH-1211, Geneva, Switzerland

(Received 5 September 2019; accepted 27 January 2020; published 10 February 2020)

We present the observation and the detailed investigation of coherent Cherenkov diffraction radiation (CChDR) in terms of spectral-angular characteristics. Electromagnetic simulations have been performed to optimize the design of a prismatic dielectric radiator and the performance of a detection system with the aim of providing longitudinal beam diagnostics. Successful experimental validations have been organized on the CLEAR and the CLARA facilities based at CERN and Daresbury laboratory respectively. With ps to sub-ps long electron bunches, the emitted radiation spectra extend up to the $\mathrm{THz}$ frequency range. Bunch length measurements based on CChDR have been compared to longitudinal bunch profiles obtained using a radio frequency deflecting cavity or coherent transition radiation (CTR). The retrieval of the temporal profile of both Gaussian and non-Gaussian bunches has also been demonstrated. The proposed detection scheme paves the way to a new kind of beam instrumentation, simple and compact for monitoring short bunches of charged particles, particularly well-adapted to novel

Published by the American Physical Society under the terms of the Creative Commons Attribution 4.0 International license. Further distribution of this work must maintain attribution to the author(s) and the published article's title, journal citation, and DOI. 
accelerator technologies, such as dielectric and plasma accelerators. Finally, CChDR could be used for generating intense $\mathrm{THz}$ radiation pulses at the MW level in existing radiation facilities, providing broader opportunities for the user community.

DOI: 10.1103/PhysRevAccelBeams.23.022802

\section{INTRODUCTION}

The emission of Cherenkov radiation by charged particles travelling through matter was discovered in 1934 [1,2] and, due to its fascinating properties (i.e., the emission of a large number of photons in a narrow and well-defined solid angle), has found numerous applications in many fields including astrophysics [3], and particle detection and identification $[4,5]$. Recently, a first experimental study was performed to demonstrate the potential of noninvasive beam diagnostic techniques based on the detection of incoherent Cherenkov diffraction radiation (ChDR) [6]. The latter refers to the emission of Cherenkov radiation by charged particles traveling not inside, but in the vicinity of a dielectric material. This combines the already well-known advantages of Cherenkov radiation with a noninvasive technique, allowing, as such, a breakthrough in beam instrumentation. The coherent emission of Cherenkov radiation by a bunch of charged particles was studied theoretically by Danos [7] in 1955, who anticipated the emission of high-power radiation at wavelengths similar or longer than the bunch length. Experimental validations came later with the development of dielectric Cherenkov masers [8] that produced high output powers in the microwave regime [9-11] using low energy $(<1 \mathrm{MeV})$, very high current $(>1 \mathrm{kA})$ electron beams with pulse durations of tens of nanoseconds. Similarly, using the backward emission of Cherenkov radiation in a metamaterial structure, high output powers were achieved by Hummelt [12] using a lower electron current $(<100 \mathrm{~A})$ and proved to be an interesting path toward compact high-power $\mathrm{THz}$ sources. Coherent Cherenkov radiation from short electron bunches was also observed experimentally in 1991 by Ciocci [13] and Ohkuma [14]. Its capability to produce high output powers at millimetre or sub-millimetre wavelengths has inspired several groups to develop dielectric wakefield acceleration (DWA) [15], where recent demonstrations have shown ultrahigh accelerating gradient (i.e., $>\mathrm{GV} / \mathrm{m}$ ) in capillary tubes [16].

To our knowledge and despite its great potential, coherent Cherenkov diffraction radiation (CChDR) has not been exploited for beam diagnostic purposes [17], and even if many groups working with short bunches have been using in the past coherent radiation, the detection systems were mainly based on coherent transition radiation (CTR) [18-22], coherent diffraction radiation (CDR) [23], coherent Smith Purcell radiation [24] or electro-optical sampling (EOS) [25].

The paper presents detailed experimental and theoretical investigations of $\mathrm{CChDR}$ spectral-angular properties.
A good consistency among electromagnetic simulations, analytic theory and experimental data obtained at two unique facilities has been achieved. The study generalizes the use of ChDR for beam diagnostics of short bunches emitting coherent radiation in a broad frequency range. The first section presents the background theory and simulation results involved for the design of a bunch length monitor. The frequency response of the radiator and its sensitivity to beam position have been worked out. The second and the third sections present the results from the experimental validation performed at two electron beam facilities, namely CLEAR at CERN and CLARA at the Daresbury laboratory. Measurements of bunch length and bunch profile have been obtained and successfully compared to simulations. We finally conclude highlighting the potentiality of such devices in existing and novel accelerators.

\section{THEORETICAL BACKGROUND}

Electrodynamics is ruled by Maxwell's equations, which describe the interaction between electromagnetic fields and matter. An electromagnetic field propagating inside or in the proximity of a given material will polarize the atoms located on its surface, giving rise to so-called polarization radiation (PR) [26]. Cherenkov diffraction radiation is a particular case of $\mathrm{PR}$ for a charged particle propagating in the vicinity of a dielectric material as shown in Fig. 1. The charge particle is characterized by its normalized velocity $\beta=v / c$ and the associated Lorentz factor $\gamma=1 / \sqrt{1-\beta^{2}}$, $c$ being the speed of light in vacuum. The radiation is

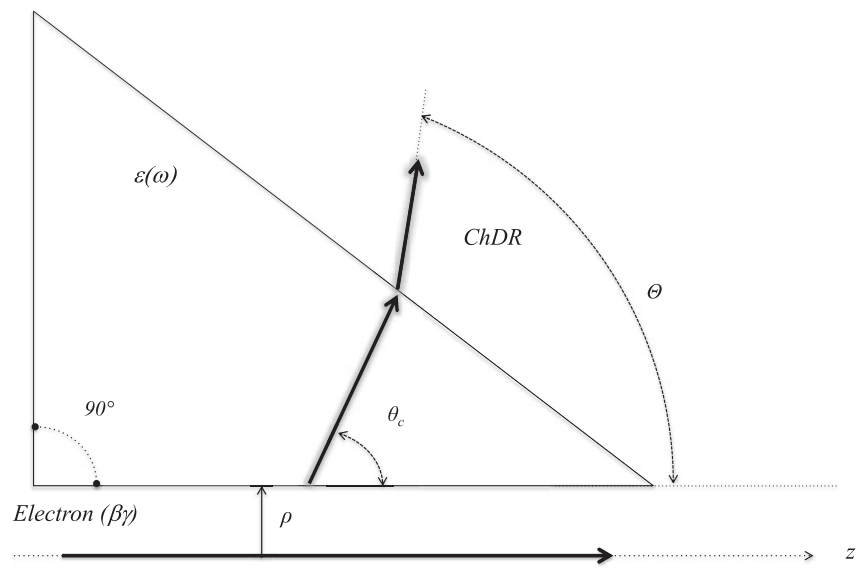

FIG. 1. Emission of Cherenkov diffraction radiation by an electron propagating at a distance $\rho$ from the surface of a conical dielectric material. 
emitted at the well-known Cherenkov angle $\theta_{c}=\arccos (1 / \beta \sqrt{\varepsilon})$, which, for relativistic particles, mainly depends on the relative permittivity of the medium, $\varepsilon$. As radiation leaves the material, the photons would naturally be refracted, following Snell's law, at an angle $\Theta$, that depends on the permittivity of the dielectric and on the orientation of the exit-face of the material (see Fig. 1). A general expression of the exact solution of Maxwell's equations for the total radiated magnetic field $\vec{H}$ can be written as:

$\vec{H}=\vec{H}_{0}-i \omega \varepsilon_{0}(\varepsilon-1) \vec{\nabla}_{\vec{r}} \times \int_{V_{R}} \Phi\left(\vec{r}-\vec{r}^{\prime}\right) \vec{E}_{0}\left(\vec{r}^{\prime}, \omega\right) d^{3} r^{\prime}$

where $\vec{E}_{0}$ and $\vec{H}_{0}$ are the electric and magnetic fields of the virtual photons associated to ultrarelativistic particles, $\varepsilon_{0}$ the vacuum dielectric constant and $\omega$ the angular frequency of the radiation. We recall that the relation between $\vec{H}_{0}$ and $\vec{E}_{0}$ is defined by:

$$
\vec{H}_{0}=\vec{v} \times \varepsilon_{0} \vec{E}_{0}
$$

The propagator $\Phi$ in Eq. (1) acts on the virtual photon's field at the position $\overrightarrow{r^{\prime}}$ radiating a photon at the position $\vec{r}$. It can be written as follows:

$$
\Phi\left(\vec{r}-\overrightarrow{r^{\prime}}\right)=-\frac{e^{i \frac{\sqrt{\varepsilon(\omega)}}{c} \omega\left|\vec{r}-\overrightarrow{r^{\prime}}\right|}}{4 \pi\left|\vec{r}-\overrightarrow{r^{\prime}}\right|}
$$

Considering the far-field condition, the ChDR magnetic field emitted within the radiator volume $V_{R}$ by a single electron, can be calculated as:

$\vec{H}-\vec{H}_{0}=\frac{\varepsilon_{0}(1-\varepsilon) \omega e^{i \frac{\sqrt{\varepsilon(\omega)} \omega}{c} R}}{4 \pi} \vec{k} \times \int_{V_{R}} \vec{E}_{0}(\vec{r}, \omega) e^{-i \vec{k} \cdot \vec{r}} d^{3} r$

where $R$ is the distance of observation. The wave vector $\vec{k}$ is defined as $\vec{k}=\sqrt{\varepsilon(\omega)} \omega \vec{R} / R c$. The observation vector is $\vec{R}=R(\cos \phi \sin \theta, \sin \phi \sin \theta, \cos \theta)$ and $\phi$ and $\theta$ are the azimuthal and polar observation angles respectively in spherical coordinates. In such a geometry, the electric field of a virtual photon is $\vec{E}_{0}$ :

$$
\vec{E}_{0}(\vec{r}, \omega)=E_{\rho}(\vec{r}, \omega) \frac{\vec{\rho}}{\rho}+E_{z}(\vec{r}, \omega) \frac{\vec{v}}{v}
$$

with the radial vector defined as $\vec{\rho}=\rho(\cos \varphi, \sin \varphi, 0)$ and the radial and longitudinal field components given by:

$$
E_{\rho}(\vec{r}, \omega)=\frac{-e \omega e^{i \frac{\omega z}{v}}}{2 \pi \gamma v^{2} \varepsilon_{0}} K_{1}\left(\frac{\omega \rho}{\gamma v}\right)
$$

$$
E_{z}(\vec{r}, \omega)=\frac{i e \omega e^{i \frac{\omega z}{v}}}{2 \pi \gamma^{2} v^{2} \varepsilon_{0}} K_{0}\left(\frac{\omega \rho}{\gamma v}\right)
$$

$K_{0}$ and $K_{1}$ are the modified Bessel functions of the second kind with zero and one order respectively. Considering the far-field ChDR radiation emitted within the radiator volume $V_{R}$ by a single electron, it is possible to show [26], processing Eq. (4), that for radiator's lengths $L$ such that $L \gg L_{f}$, where $L_{f}$ is the formation length of the ChDR inside the volume $V_{R}$ :

$$
L_{f}=\left|\frac{v}{\omega(1-\beta \sqrt{\varepsilon(\omega)} \cos \theta)}\right|
$$

the angular-spectral distribution of the radiated energy can be expressed as:

$$
\frac{d^{2} I}{d \Omega d \omega} \bar{\sim}\left|f\left(V_{R}, \omega\right)\right|^{2} \frac{e^{2} \omega L}{8 \pi^{2} \varepsilon_{0} c^{2}} e^{-2 \frac{\omega \omega}{\gamma c}} \delta\left(\theta-\theta_{c}\right)
$$

where $f$ is a Fraunhofer far-field-diffraction term, depending on the radiator shape and on the emitted photon energy, $d \Omega=\sin \theta d \theta d \phi$ is the unitary solid angle of radiation collection and where the electron impact parameter, i.e., the distance between the electron and the dielectric surface, has been denoted by $a$.

\section{ELECTROMAGNETIC SIMULATIONS}

A detailed simulation study has been initiated in order to investigate the expected performance and limitation of a detection system based on CChDR for short bunch length monitoring.

\section{A. Simulations with MAGIC}

2D axi-symmetric simulations have been first performed using the MAGIC code [27] studying the emission of CChDR in a hollow conical dielectric radiator with an opening angle of $45^{\circ}$. For this purpose, simulations have been running using an hypothetical $1.2 \mathrm{~ns}$ long electron bunch with a time dependent sinusoidal current modulation at a given frequency. Figure 2 shows examples of CChDR emitted by a hollow cone with an internal radius of $0.5 \mathrm{~mm}$ by an electron beam with an average current electron of $1 \mathrm{~A}$ modulated at a frequency of $25 \mathrm{GHz}$. The two cases displayed are assuming dielectric with a different relative permittivity. The photons leave the radiator with a different angle, at $90^{\circ}$ for $\varepsilon=5$ and $45^{\circ}$ for $\varepsilon=2.1$ as shown in Figs. 2(a) and 2(b) respectively. In the second case, the Cherenkov emission angle inside the dielectric is already at $45^{\circ}$ and the radiation leaves the material straight through. The amplitude of the magnetic field recorded at the point $P$ located $10 \mathrm{~cm}$ away from the exit face of the dielectric is also depicted in Fig. 3 for completeness. In order to study the frequency response and the position sensitivity of such 


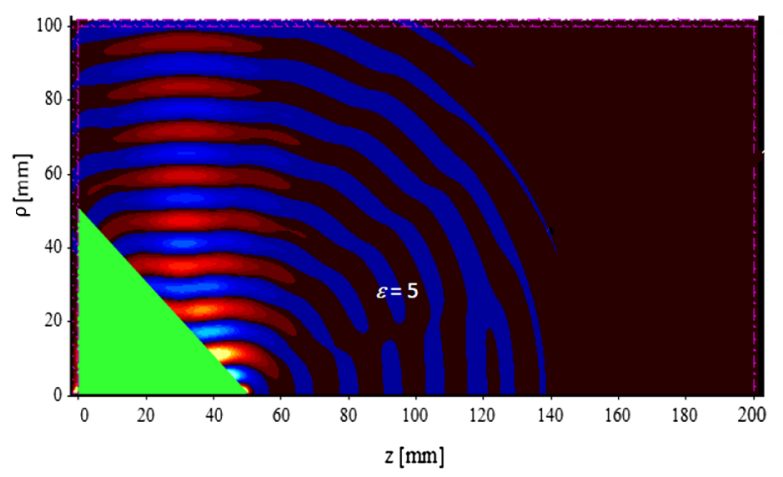

(a)
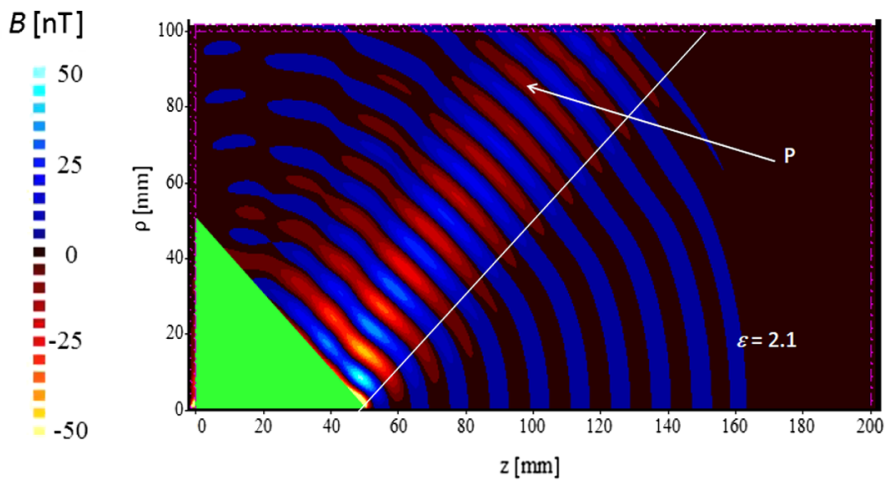

(b)

FIG. 2. Simulations performed using MAGIC [27] of CChDR emitted by a $0.5 \mathrm{~mm}$ radius hollow dielectric cone. (a): with a relative permittivity $\varepsilon=5$. (b): with a relative permittivity $\varepsilon=2.1$.

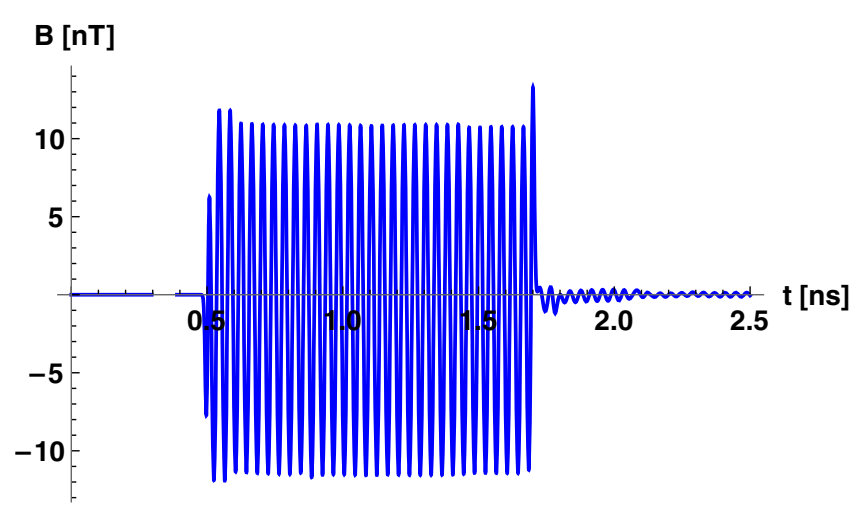

FIG. 3. Time domain magnetic field of the radiation recorded at a point $P$ visible of Fig. 2(b) and located $10 \mathrm{~cm}$ away from the exit face of the dielectric.

a monitor, a series of simulations were performed, using a radiator made of PolyTetraFluoroEthylene (PTFE) that has an $\varepsilon=2.1$ in the sub- $\mathrm{THz}$ region. A first series has been done varying the frequency of the electron beam modulation from 6.25 to $100 \mathrm{GHz}$. For each of those modulation frequency, simulations have also been run using hollow dielectric cones with different internal radii ranging from 0.5 to $5 \mathrm{~mm}$, while considering an electron beam passing through the center of the hollow channel. For each simulation case, the output power recorded at point $P$ has been extracted. The results have been then normalized to the maximum output power and plotted as a relative transfer function of the radiator, which corresponds to the spectrum ideally emitted by a single particle. Figure 4(a) shows the transfer function for different internal radii $\varrho$ of the hollow conic dielectric while the frequency response of the radiator is presented Fig. 4(b). Each of the points in Fig. 4(b) is the average of the transfer functions obtained from the simulation of hollow cone with different internal radii. Both plots show an excellent agreement between simulations obtained with MAGIC and calculations using the analytic model reported in Refs. [28], based on the formulas given in the previous section. The photon intensity decreases at lower frequencies as electromagnetic oscillations at large wavelengths compared to the physical

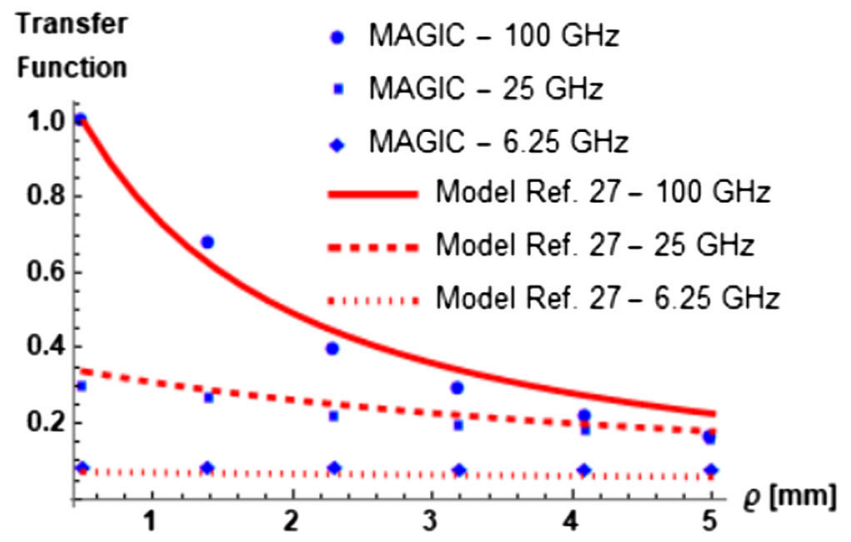

(a)

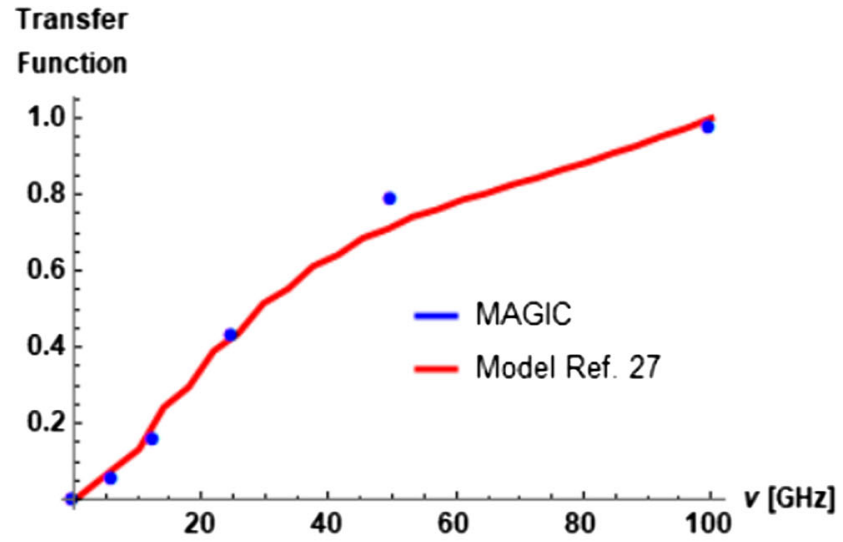

(b)

FIG. 4. (a): Transfer function of conical radiator $(\varepsilon=2.1$ ) with internal radius $Q$ ranging from $0.5 \mathrm{~mm}$ to $5 \mathrm{~mm}$. (b): Transfer function of the hollow conical radiator $(\varepsilon=2.1)$ as a function the radiated frequency. 
TABLE I. Simulation parameters.

\begin{tabular}{lc}
\hline \hline Parameter & Value \\
\hline Bunch transverse size (rms) & $0.9 \mathrm{~mm}$ \\
Bunch duration (rms) & $1-5 \mathrm{ps}$ \\
Bunch charge & $100 \mathrm{pC}$ \\
Dielectric permittivity (teflon) & 2.1 \\
\hline \hline
\end{tabular}

size of the radiator cannot be generated and propagated inside the medium. The size of the radiator can thus be optimized to provide a detection system with an adequate frequency response adapted to specific needs. For all frequencies the emitted power decreases when increasing internal radius. However, as expected from diffraction radiation theory, the output power decreases significantly faster for higher frequencies. One can observe a reduction by a factor 8 and 2 for frequencies of $100 \mathrm{GHz}$ and $25 \mathrm{GHz}$ respectively. In a given detector geometry, one can then expect a very different sensitivity response to beam position offset depending on the measured frequency. This is also an interesting and crucial aspect of CChDR that should be taken into account when designing and using detectors based on it.

\section{B. Simulations with VSim}

In accelerators, charged particles are typically grouped in short bunches that would emit coherent radiation in a broad frequency range. Simulations with such a short electron bunch have been made using the VSim particle-in-cell solver [29] with beam parameters presented in Table I similar to those available in facilities for which we report experimental results later in the paper. The radiator made of
PTFE has been considered having an internal radius of $5 \mathrm{~mm}$. Instead of simulating the beam as a bunch of macroparticles, the electron beam has been simulated longitudinally and transversely as a Gaussian distributed charge. This helps minimizing numerical noise caused by the mesh size and its limited resolution at high frequencies [30]. Two examples are shown in Fig. 5 with electron beam propagation on-axis [Fig. 5(a)] and at an offset of $2 \mathrm{~mm}$ [Fig. 5(b)]. As shown in the previous section, the CChDR wave-front generated inside the dielectric propagates at $45^{\circ}$ and exits the dielectric straight through. In order to calculate the coherent power spectrum produced in the radiator, the transverse electric field generated in Vsim has been recorded as a function of time at one of the detector's location (see Fig. 5) and then Fourier-transformed. This procedure has been repeated for five different rms bunch lengths, from 1 to $5 \mathrm{ps}$. The reconstructed CChDR spectra are depicted in Fig. 6 for an electron bunch traveling on axis within the hollow channel of the dielectric prism, compared to the CChDR spectra calculated via the model of Ref. [28] in the same conditions.

\section{EXPERIMENTS AT CLEAR}

The CERN Linear Electron Accelerator for Research (CLEAR) [32] exploits $\lesssim 1 \mathrm{ps,} \sim 200 \mathrm{MeV}$ electron bunches for several applications including the development of novel concepts for $\mathrm{THz}$ generation and beam diagnostics [33]. A layout of the accelerator facility and the experimental setup is presented in Fig. 7, while the beam parameters are summarized by Table II. The electron beam is generated on a Cs2Te photo-cathode $(\mathrm{QE}>0.3 \%$, lifetime 1 year) using an UV (converted from IR) laser.

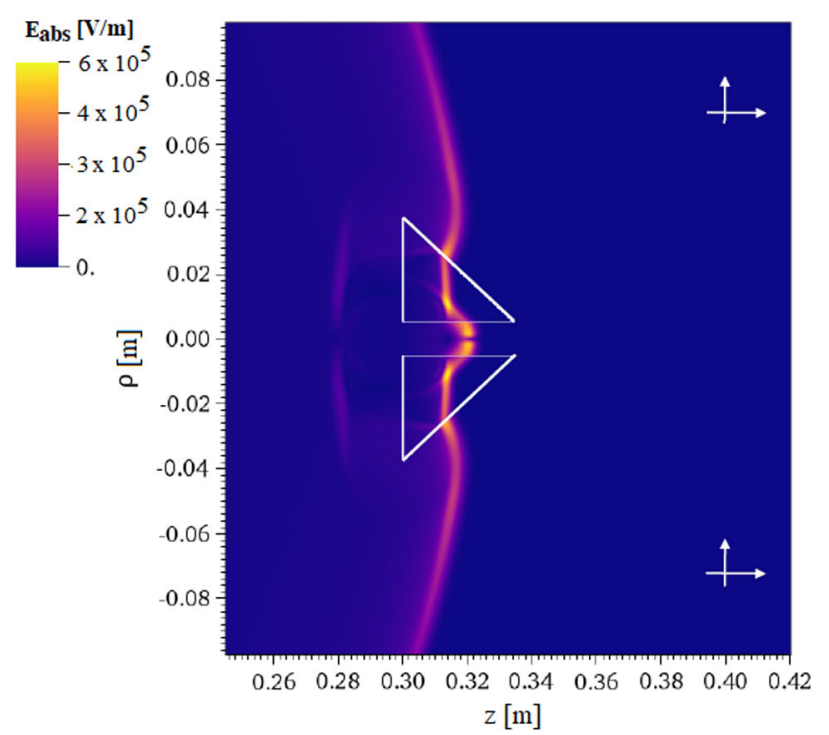

(a)

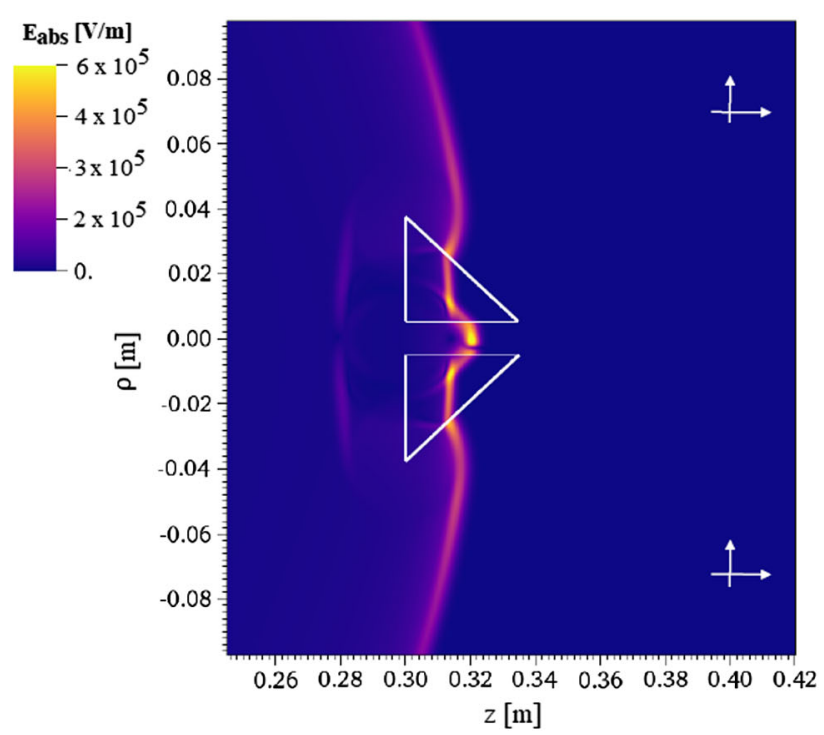

(b)

FIG. 5. Simulations of electron bunch propagation in the hollow channel of the dielectric prism for a on-axis (a) and a off-axis (b) beam. Detectors positions in the propagation plane are marked by white axes. 


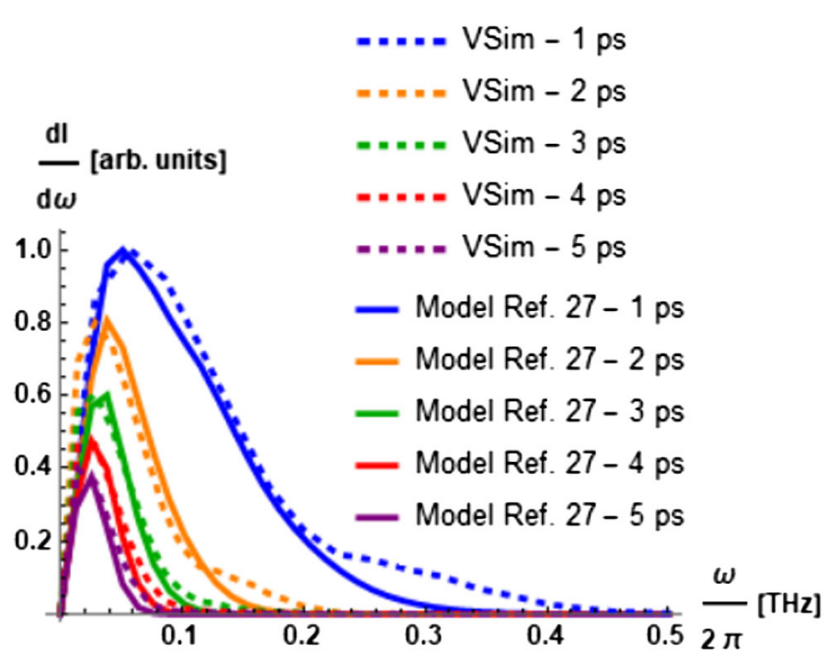

FIG. 6. CChDR spectra simulated using VSim for different electron bunch lengths compared to the CChDR spectra calculated via the model in Refs. [28,31].

The rf-gun is followed by three $4.5 \mathrm{~m}$-long, $3 \mathrm{GHz}$ accelerating structures. The first structure can be used as a buncher to tune the bunch length from $0.5 \mathrm{ps}$ to $10 \mathrm{ps} \mathrm{rms}$, by means of velocity bunching. A S-band rf deflector is installed just downstream of the accelerating structures for bunch length diagnostics [32]. The transverse mode propagating inside this cavity provides a time dependent beam deflection, mapping the longitudinal distribution of the electron bunches into a transverse profile monitored by a scintillator screen positioned downstream (top-right inset of Fig. 7). When the rf deflector is off, the beam can be transported straight to the end of the beam line where a $\sim 1.5 \mathrm{~m}$ long in-air experimental test stand is located. For our present studies, a dielectric radiator has been installed together with the associated detectors as shown in Fig. 7 (bottom-right inset). The radiator is a $2.5 \mathrm{~cm}$ long hollow prism made out of PTFE with a $5 \mathrm{~mm}$ internal radius and a $5 \mathrm{~cm}$ wide base. The geometry of the detection system has been chosen in order to be sensitive to bunch length variations. Three of the four output faces of the prism are instrumented using horns and waveguides in V, W, and G bands to collect and measure ChDR in three independent frequency bands. Each detection line is composed of a rectangular waveguide horn, band-pass filters and Schottky diodes from Millitech. The two detectors installed on the left and right sides of the dielectric are measuring at frequencies of $84 \pm 1 \mathrm{GHz}$ and $113.5 \pm 9 \mathrm{GHz}$ respectively. The third detector placed in a plane $45^{\circ}$ upward is detecting radiation emitted at a frequency of $60 \pm 1 \mathrm{GHz}$. The gain-horns have an aperture of $4 \times 4 \mathrm{~cm}^{2}$ and $3 \times$ $2 \mathrm{~cm}^{2}$ for the 60 and $84 \mathrm{GHz}$ detection lines respectively and $2 \times 1.5 \mathrm{~cm}^{2}$ for the $113.5 \mathrm{GHz}$ one. The relative sensitivity of the three detection lines is taken into account when analyzing the experimental data, including the response curves of the Schottky diodes, the bandwidth of the band-pass filters and the gain of the horns.

\section{A. Bunch length monitoring with coherent Cherenkov diffraction radiation}

The temporal profile of the photocathode laser beam is typically Gaussian, nevertheless by adjusting the amplitude

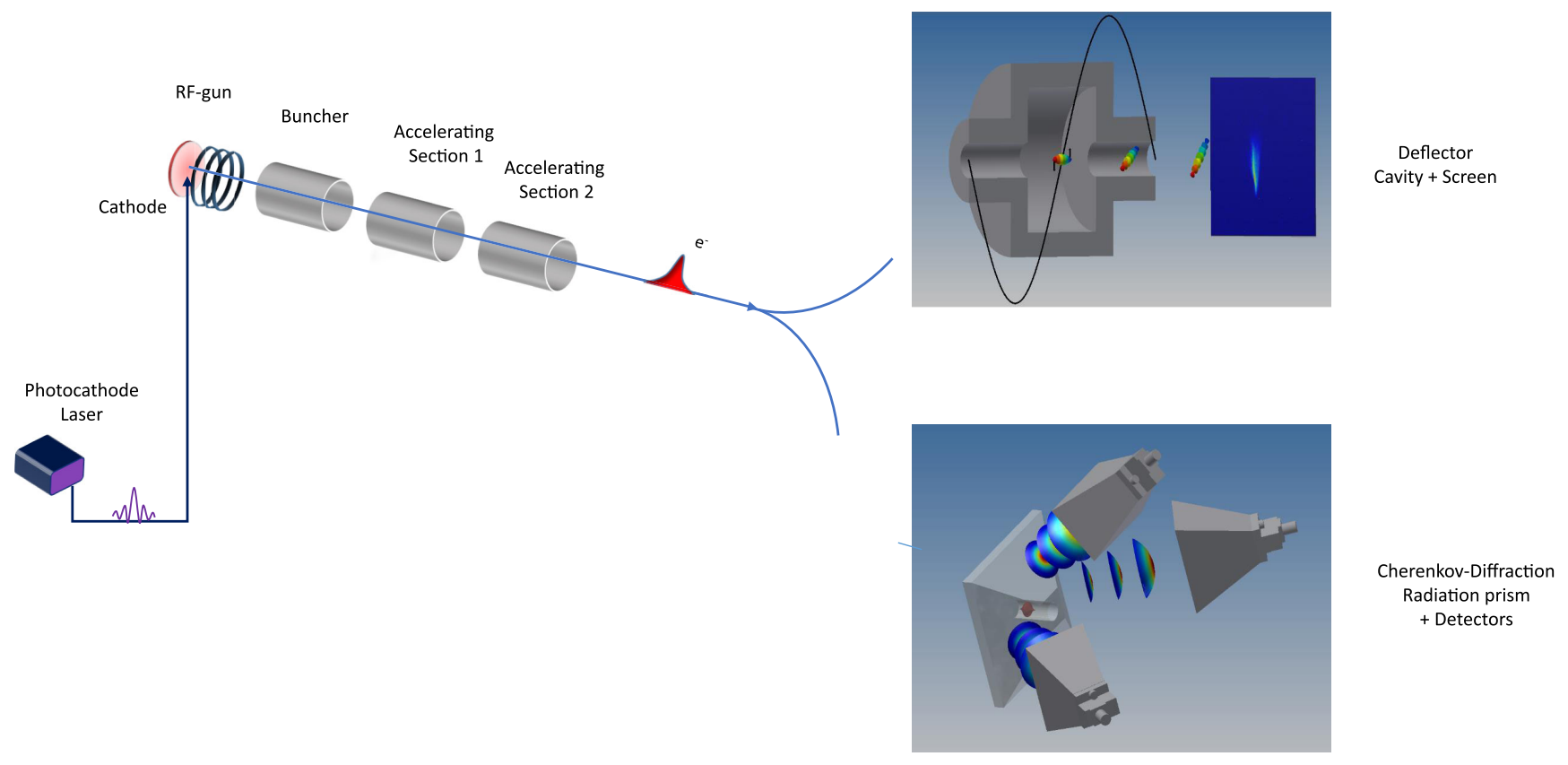

FIG. 7. Sketch of the CLEAR beam line featuring with a rf-deflecting cavity used for longitudinal profile measurement and a dielectric prism made out of PFTE. The CChDR is detected in three independent frequency bands using horns and waveguides. The machine beam parameters are also reported in the table. 
TABLE II. Beam parameters of CLEAR.

\begin{tabular}{lc}
\hline \hline Beam parameter & Value range \\
\hline Beam energy $[\mathrm{MeV}]$ & $50-220$ \\
Bunch charge $[\mathrm{nC}]$ & $0.001-1.5$ \\
Bunch length rms [ps] & $0.5-10$ \\
Relative energy spread (rms) & $<0.2 \%$ \\
Frequency of the electron pulse [GHz] & 1.5 \\
Number of bunches in a pulse & $1-200$ \\
Repetition rate of the electron pulse $[\mathrm{Hz}]$ & $0.833-10$ \\
\hline \hline
\end{tabular}

and phase of the rf signal injected in the rf gun, one can modify the longitudinal shape of the electron bunch and produce Gaussian and skew-Gaussian bunch shapes. Bunch length measurements performed using CChDR have been compared to the longitudinal beam profile obtained using the rf deflecting cavity, which can resolve bunch profiles as short as $\sim 100 \mathrm{fs}$ rms. A typical measurement with the rf deflector is based on the average of ten consecutive shots in order to minimize the importance of statistical fluctuations.

\section{Alignment procedure of the beam inside the prism}

Both the ChDR prism and the Schottky diode detectors are mounted on a remotely controlled and adjustable optical table driven by stepping motors that allow to displace the experimental setup both in the horizontal and vertical planes independently. Initially the center of the hollow prism has been precisely positioned to the beam axis using laser alignment. A 1.5 ps long electron bunch has been sent through the center of the radiator, focused down to $\lesssim 1 \mathrm{~mm}$ rms transverse beam size, and kept stable in the same configuration during the measurements. In order to align the beam in the center of the ChDR detector, the whole experimental setup has then been moved horizontally by steps of $\sim 200 \mu \mathrm{m}$, the corresponding radiation power being recorded using the two horizontal detectors. The experimental results are presented in Fig. 8 that shows

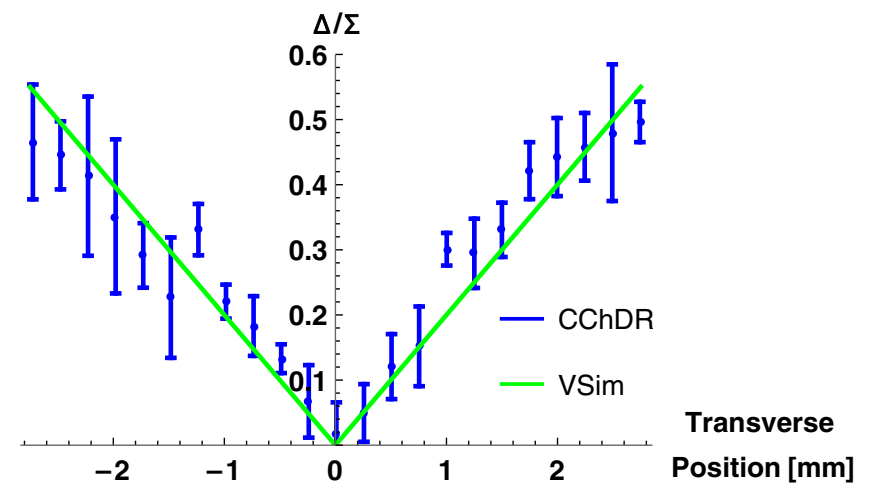

FIG. 8. Beam position signal reconstructed using CChDR, compared to simulations. Experimental points obtained averaging over 10 pulses, each containing 10 electron bunches. the evolution (as a function of the stepper motor position) of the reconstructed beam position signal, which is calculated as the absolute value of difference over the sum $\Delta / \Sigma$ of the signals coming from the two horizontal detectors. With a transverse beam size of $1 \mathrm{~mm}$, the scan in position cannot be performed over a range larger than $\pm 2.5 \mathrm{~mm}$, which is half of the whole aperture. For larger position offsets, the direct emission of Cherenkov radiation, produced by transverse beam tails passing through the dielectric itself, distorts the measurements. Simulation results using Vsim are also presented on the same plot for comparison. The electric field obtained by simulations is recorded at the two locations, left and right from the prism output surface, as presented in Fig. 5. For each beam position offset, the time dependent fields are first squared and integrated in order to obtain the expected CChDR power from both sides of the radiator. The simulation results are also plotted as the absolute value of difference over sum of the two corresponding output powers, similarly to what is done experimentally. The comparison between experiments and simulations clearly shows a very similar trend. The present configuration has provided a practical way to center the beam inside the prism, which has been a prerequisite in order to provide reliable bunch length measurements as they are presented in the following.

\section{Measuring Gaussian electron bunches}

The coherent emission from a Gaussian electron bunch, defined by its rms bunch length $\sigma_{\tau}$, is obtained by introducing the quantity $\left|j\left(\omega, \sigma_{\tau}\right)\right|^{2}=2 \pi j_{0}^{2} \sigma_{\tau}^{2} \exp \left(-\omega^{2} \sigma_{\tau}^{2}\right)$ (also called bunch form factor), which multiplies Eq. (9):

$$
\frac{d^{2} I}{d \Omega d \omega} \bar{\sim}\left|f^{(0)}\right|^{2} j_{0}^{2} \sigma_{\tau}^{2} e^{-\left(\omega^{2} \sigma_{\tau}^{2}+\frac{2 \omega a}{\gamma c}\right)} \frac{e^{2} \omega L}{4 \pi \varepsilon_{0} c^{2}} \delta\left(\theta-\theta_{c}\right)
$$

where $f^{(0)}=f^{(0)}\left(V_{R}\right)$. Considering independent measurements performed at two similar angular frequencies $\omega_{1} \sim \omega_{2}$, the geometrical factor $f$ in Eq. (9) assumes approximately the same value upon those frequencies. According to the transfer function in Fig. 4(b) and to the frequencies adopted for the experiment, the relative error made with the statement $f\left(V_{R}, \omega_{1}\right)=f\left(V_{R}, \omega_{2}\right)=f^{(0)}$ is $\sim 10 \%$. The intensity ratio $S_{1} / S_{2}$, corresponding to the signals $S_{1}=S_{1}\left(\omega_{1}\right)$ and $S_{2}=S_{2}\left(\omega_{2}\right)$ measured at $\omega_{1}$ and $\omega_{2}$, each of them proportional to the quantity defined above as $d^{2} I / d \Omega d \omega$, can be written via Eq. (10) as:

$$
\frac{S_{1}}{S_{2}}=e^{-\left(\omega_{1}^{2}-\omega_{2}^{2}\right) \sigma_{\tau}^{2}} \frac{\omega_{1}}{\omega_{2}} e^{-\frac{2\left(\omega_{1}-\omega_{2}\right) a}{\gamma c}}
$$

The rms bunch length $\sigma_{\tau}$ can thus be obtained as a function of $S_{1}$ and $S_{2}$ : 


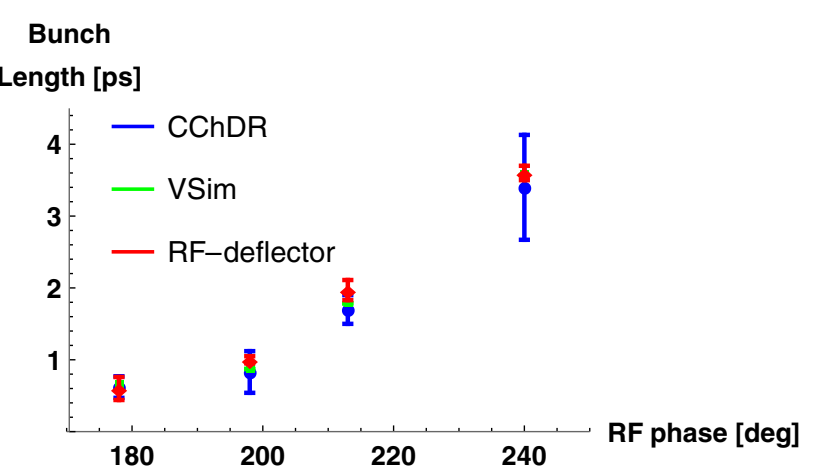

(a)

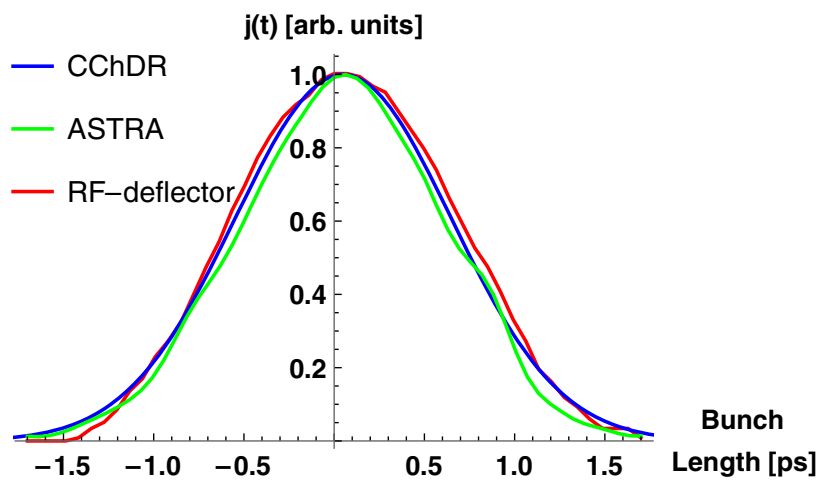

(b)

FIG. 9. Top figure (a): Direct comparison between the rms bunch length of Gaussian bunches as measured with the rfdeflector and by detection of CChDR. Simulation results are also reported. Bottom figure (b): Comparison among bunch profiles obtained with CChDR, with rf-deflector and simulated with ASTRA, corresponding to the bunch length $\sim 0.6$ ps rms in Fig. 9(a).

$$
\sigma_{\tau}=\sqrt{\left|\frac{1}{\omega_{1}^{2}-\omega_{2}^{2}}\left[\log \left(\frac{S_{1} \omega_{2}}{S_{2} \omega_{1}}\right)+\frac{2\left(\omega_{1}-\omega_{2}\right) a}{\gamma c}\right]\right|}
$$

The difference in the detection bandwidths between $\Delta \omega_{1}$ and $\Delta \omega_{2}$ is not contained within Eq. (12), therefore it has been taken into account as a systematic error on the bunch length measurement $d \sigma_{\tau}=\left(d \sigma_{\tau} / d \omega_{1}\right) \Delta \omega_{1}+$ $\left(d \sigma_{\tau} / d \omega_{2}\right) \Delta \omega_{2}$. Figure 9 presents a comparison between rf-deflector and CChDR measurements as a function of the rf phase of the electron gun. In this case, Gaussian bunches with a $100 \mathrm{pC}$ charge have been used. The agreement is excellent for short bunches and the larger error visible on the CChDR measurements for longer bunches is due to the weakness of the corresponding power emitted in the detection frequency bands (i.e., $84 \mathrm{GHz}$ and 113.5 GHz) for such bunch lengths. This could be overcome by choosing detectors working at lower frequencies. Figure 9(a) also includes a comparison with electromagnetic simulations performed with VSim. From the simulated bunch power spectrum shown in Fig. 6, we computed and plotted the simulated power ratio of $S(84 \mathrm{GHz}) / S(113.5 \mathrm{GHz})$. The agreement is within $5 \%$ and it clearly highlights the validity of our approach. The comparison between the longitudinal profile measured with the rf deflector and the one reconstructed from CChDR is shown in Fig. 9(b) for completeness, compared to ASTRA simulations [34].

\section{Measuring skew-Gaussian electron bunches}

Skew-Gaussian bunch profiles have been also produced on CLEAR by exploiting an energy-time correlation along the temporal profile of the electron bunch, adjusting the phase of the rf gun. This effect depends strongly on the bunch charge and has been studied in depth using ASTRA simulations [34]. Negligible below a bunch charge between 200 and $300 \mathrm{pC}$, it becomes important for charges around $400 \mathrm{pC}$, as the one used for the experiment shown in this section. At even higher charges, space-charge effects would produce super-Gaussian longitudinal profiles. In order to detect skew-Gaussian bunch profiles, a different model, as reported in the Appendix, must be used. This extracts both the bunch length and the skewness parameters, $\sigma_{\tau}$ and $\alpha$ respectively. An additional measurement point at a different frequency is however necessary in order to extract both parameters. Experimentally, data from the third diode $S_{3}$ working at $60 \pm 1 \mathrm{GHz}$, have been taken into account by numerically solving the following system of equations:

$$
\begin{aligned}
& \frac{S_{1}}{S_{2}}=\frac{\left|j\left(\omega_{1}, \sigma_{\tau}, \alpha\right)\right|^{2}}{\left|j\left(\omega_{2}, \sigma_{\tau}, \alpha\right)\right|^{2}} \frac{\omega_{1}}{\omega_{2}} e^{-\frac{2\left(\omega_{1}-\omega_{2}\right) a}{\gamma c}} \\
& \frac{S_{1}}{S_{3}}=\frac{\left|j\left(\omega_{1}, \sigma_{\tau}, \alpha\right)\right|^{2}}{\left|j\left(\omega_{3}, \sigma_{\tau}, \alpha\right)\right|^{2}} \frac{\omega_{1}}{\omega_{3}} e^{-\frac{2\left(\omega_{1}-\omega_{3}\right) a}{\gamma c}}
\end{aligned}
$$

The expression of the bunch form factor for the skewGaussian case is calculated explicitly within Appendix. A comparison between measurements and simulations performed by ASTRA is presented in Fig. 10, showing a good agreement among the methods.

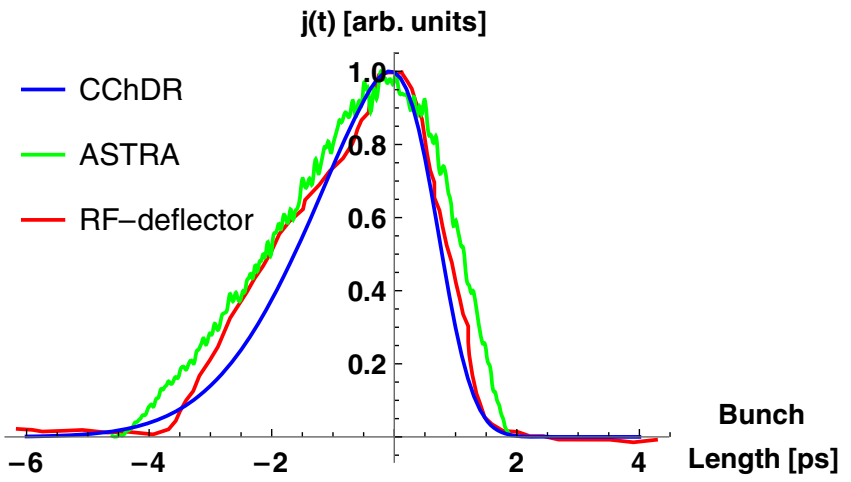

FIG. 10. Skew-Gaussian bunch profile measured with rf-deflector and CChDR, then simulated by ASTRA. 


\section{EXPERIMENTS AT CLARA}

The experimental work presented in this section has been conducted at CLARA/VELA facility in Daresbury Laboratory-STFC [35]. The CLARA photoinjector generates electron beams with an energy $35 \mathrm{MeV}$ at $10 \mathrm{~Hz}$ bunch repetition frequency, with charge ranging from 70 to $100 \mathrm{pC}$. The beams are then injected into the VELA beam line where the bunches can be compressed from 2 ps to $0.2 \mathrm{ps}$ rms by varying the phase of an accelerating cavity, similarly as done at CLEAR. The beams are then delivered to the so-called $B A 1$ experimental zone, where a vacuum chamber can host devices under test.

\section{A. Experimental setup}

For the CChDR measurements this chamber has been equipped with a remotely controlled manipulation system and a suite of beam diagnostics including an energy spectrometer, YAG screens, and beam position monitors. In the final setup, shown in Fig. 11, a CChDR and a CTR radiator have been installed in the experimental vacuum chamber with the aim to compare their radiation spectra and their capability to measure short bunch lengths. The CChDR radiator has been a triangular prism made of PTFE with a base size of $5 \mathrm{~cm}$ and $4 \mathrm{~cm}$ width, while the CTR screen has been a $2 \mathrm{~cm}$ radius metallic (polished steel) screen $2 \mathrm{~mm}$ thick. Both radiators have been installed on individual holders, each of them mounted on a movable platform with three degrees of freedom. When using CTR, the beam has been steered through the center of the screen, while in the case of CChDR, the distance between the beam

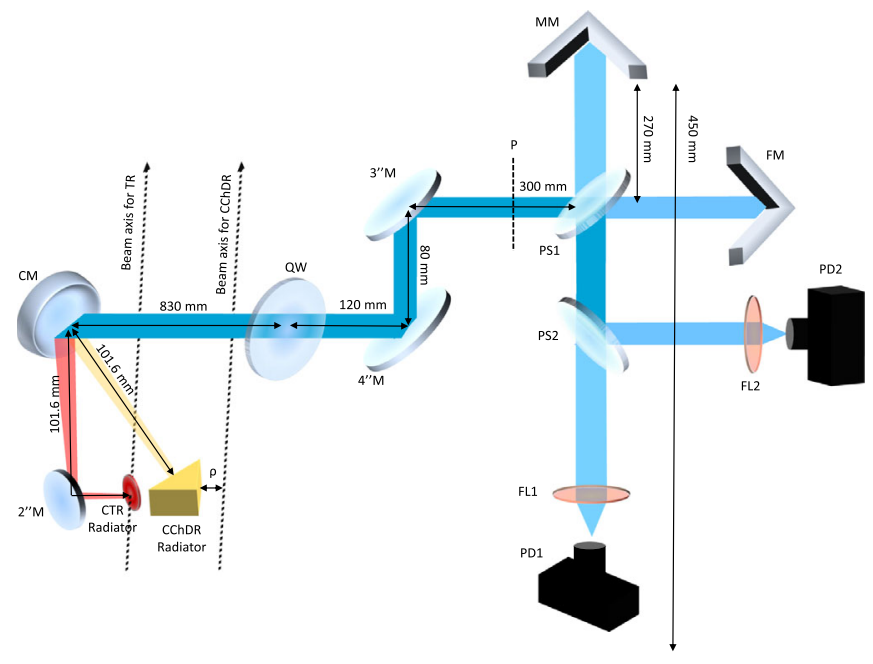

FIG. 11. Experimental setup for CChDR and CTR extraction and interferometry: CM-Concave Mirror; QW-Quartz Window; 4 inchM -4 inches Mirror; 3 inchM-3 inches Mirror; PPolarizer; PS1, PS2-Polarizing beam Splitters; FM-Fixed Mirror, MM-Movable Mirror; PS2_Polarizing beam Splitter; FL1, FL2-Focusing Lenses with focal length 101.6 mm; PD1, PD2-Pyroelectric Detectors (Gentec THz51). and the surface of the dielectric has been typically adjusted between 0.1 and $2 \mathrm{~mm}$. The generated radiation has been reflected by an in-vacuum concave mirror with a focal length of $101.6 \mathrm{~mm}$, installed on a rotating platform. The distance between the mirror and the radiators has been set to be equal to its focal length in such a way that the radiation was parallel over long distances (see Fig. 11). The rotating platform has been mounted on a translational stage enabling to adjust the position of the concave mirror when moving the CChDR radiator. The radiation has been then extracted through a quartz window and analyzed using a Martin-Puplet interferometer [36], by means of a system of motorized mirrors. The Martin-Puplett interferometer is one of the most efficient instruments to measure radiation spectra in (sub-) THz frequency range. Due to its design with a polarizing beam splitter, it provides the ability to eliminate the influence of internal interference and the effect of the charge fluctuations in the accelerator, using two detectors (PD1 and PD2 in Fig. 11). The signals obtained with these two detectors are anticorrelated relative to each other. Therefore, if dividing the signal difference by the sum, a normalized interferogram $\delta(x)$ is obtained as:

$$
\delta(x)=\frac{I_{P D 1}-I_{P D 2}}{I_{P D 1}+I_{P D 2}}
$$

The $x$ variable here is the difference in length between the two arms of the interferometer. This procedure has required a fine alignment of all the elements of the interferometer. The alignment has been performed using a test $\mathrm{THz}$ source and a new-line Terasence camera operating from $10 \mathrm{GHz}$ to $1 \mathrm{THz}[37]$.

\section{B. Bunch length reconstruction with coherent Cherenkov diffraction radiation}

Interferograms of CChDR and CTR registered at the beam energy of $35 \mathrm{MeV}$, the beam charge of $70 \mathrm{pC}$, and for the impact parameter of $1 \mathrm{~mm}$ are shown in Figs. 12(a) and 12(b). The data acquisition system has been synchronized with the accelerator rf system. Fifty samples from each pyroelectric detector for each step of the scanning interferometer mirror have been recorded to minimize the statistical uncertainty. Interferograms are shown with a triangular apodization window [38]. No other postprocessing techniques, including zero-padding have been used. Figures 12(c) and 12(d) show the result of Fourier transform of $\delta(x)$ and corresponding single electron spectra. For single electron spectra, the models described in [28] for CChDR and [31] for CTR have been used. In particular, the CChDR radiation model takes into account the beam energy, radiator geometry, impact parameter, relative position between collecting optics and radiator, angle between radiator and beam-axis, and radiator refractive index. At lower frequencies both spectra decrease in intensity due to finite radiator dimensions and diffraction effects in the 

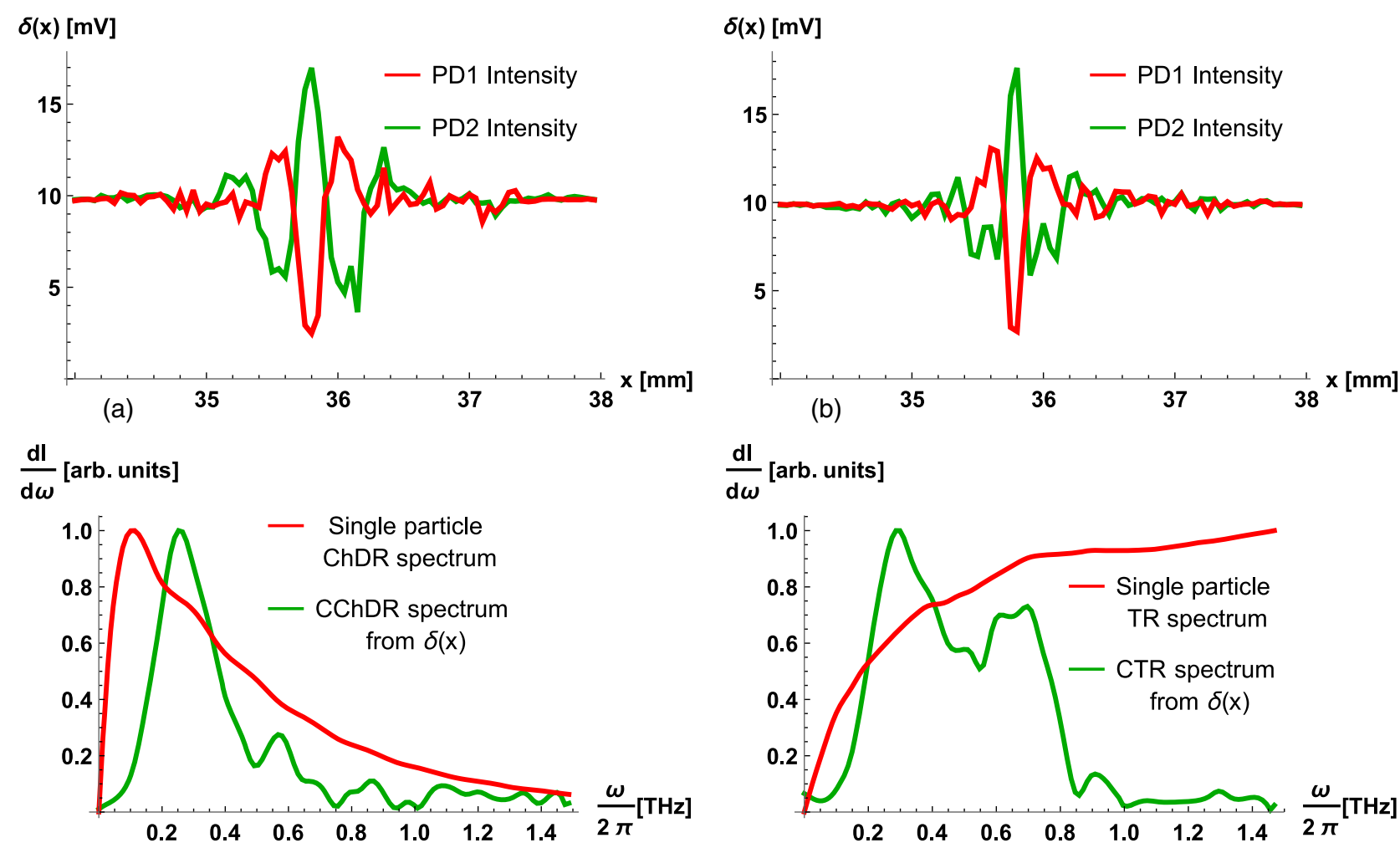

(c)

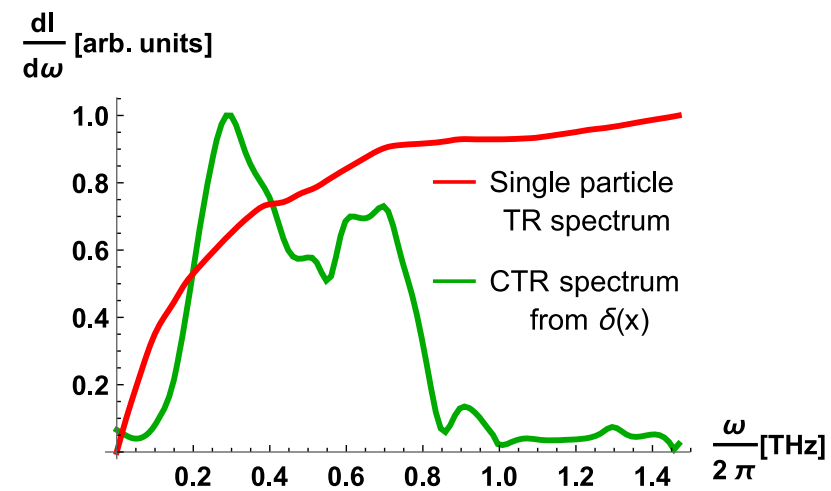

(d)

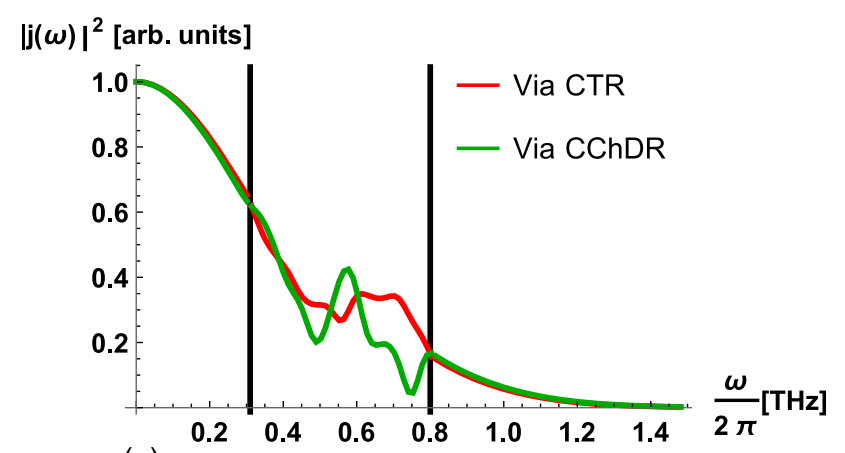

(e)

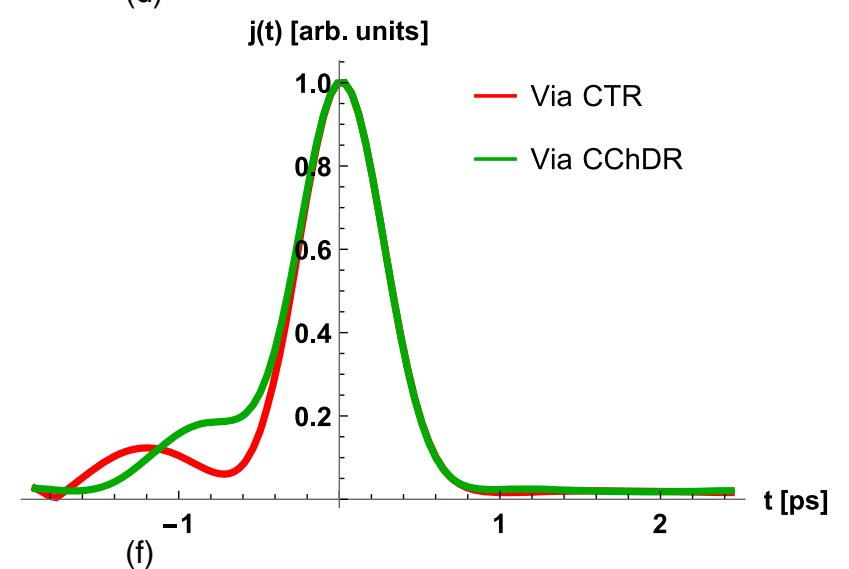

FIG. 12. Interferograms of the CChDR (a) and CTR (b) measured by two detectors; single electron (red) and spectra of CChDR (c) and CTR (d) derived from the corresponding interferograms; (e) extrapolated bunch form factor via both CChDR and CTR; (f) bunch profile reconstruction via CChDR and CTR given the same rf settings in the accelerator.

radiation delivery system. At higher frequencies the spectra are dominated by the electron bunch length and longitudinal profile modulations. The longitudinal bunch form factor has been derived normalizing the radiation spectra by single electron spectra. Figure 12(e) illustrates the comparison between the bunch form factors extracted from CTR and CChDR spectra. For the data analysis we have chosen the central part of the form factor from 300 to $800 \mathrm{GHz}$. The low frequency part $(<300 \mathrm{GHz})$ is distorted by the diffraction effects caused by concave mirror, output quartz view port, finite apertures of the interferometer, motorized mirrors and detector. These effects are not taken into account in the single electron spectrum and, therefore cannot be used for analysis. At higher frequencies the coherent radiation is significantly suppressed by the bunch length. In this case the apparatus noise generates artifacts in the analysis which are significantly different for CChDR and CTR. Therefore both curves have been extrapolated to lower and higher frequencies from the same points by a Gaussian and an exponential curve, following the prescriptions in [39]. At the connection points the curve value and its first derivative were set to be equal. The extrapolated curves are shown in 12(e), outside the region limited by the black lines placed at the connection points. 
The reconstruction of the bunch temporal profile by just an inverse Fourier transform is not possible because only the amplitude of $j(\omega)$ can be measured in an experiment, but not its phase. According to [40], the amplitude $|j(\omega)|$ and the phase $\psi(\omega)=\arg (j(\omega))$ are related by the KramersKronig relation in a way that if the function $|j(\omega)|$ is measured at all frequencies, then the phase $\psi(\omega)$ is obtained as:

$$
\psi(\omega)=-\frac{2 \omega}{\pi} \int_{0}^{\infty} \frac{\ln (|j(w)| /|j(\omega)|)}{w^{2}-\omega^{2}} d w
$$

The longitudinal bunch profile can be derived as:

$$
j(t)=\frac{1}{\pi c} \int_{0}^{\infty}|j(\omega)| \cos (\psi(\omega)-\omega t) d \omega
$$

For the analysis the integrals have been replaced by fast Fourier transform (FFT) algorithms. The frequency range has been chosen from zero to the point beyond which the phase does not make any difference. Figure 12(f) shows the bunch profile reconstruction taking into account the phase. The full width at half maximum (FWHM) has been measured to be $686 \pm 14 \mathrm{fs}$ with CChDR and $640 \pm$ 12 fs with CTR. Such a result is in agreement with the expected value of bunch length for this machine. Despite a serious difference in CChDR and CTR spectra due to differences in the single electron spectra, after normalization the longitudinal form factors look very similar. The reconstructed bunch profiles are shown in 12(f) demonstrate a good consistency of the cores of the distributions. However the tails are different, formed from instabilities in the bunch compression configuration and might vary shot-by-shot. To measure the tails precisely, a single shot spectrometer system is needed. The current setup has enabled us only to diagnose a stable part of the beam. Typical bunch length measurements, including interferometry, FFT, single electron spectrum normalization, and Kramers-Kronig reconstruction have been taking approximately fifteen minutes. Nevertheless, this time can be reduced down to one minute by using detectors with higher signal to noise ratio and an automatic software-based procedure.

\section{CONCLUSIONS}

We have presented a detailed theoretical and experimental investigation of coherent Cherenkov diffraction radiation (CChDR) spectral-angular characteristics. We have demonstrated how electromagnetic simulations match the analytical theory. The experimental verification performed on two independent electron beam accelerator test facilities, namely on the CLEAR at CERN and on the CLARA at the Daresbury laboratory, is in excellent agreement with theoretical expectations, confirming its key unique properties, e.g., high directionality, intense photon yield and noninvasive nature. The feasibility of the CChDR phenomenon as a tool for non-invasive short bunch diagnostics has been investigated. Supported by electromagnetic simulations, we have demonstrated that the bunch length can be retrieved using a simple and cost-effective technical solution. Bunches in the sub-ps to ps range have been measured, successfully benchmarking this new detection scheme with more standard but invasive techniques, e.g., rf deflecting cavities or coherent transition radiation. The simulations made in VSim have shown that the wakefields' pattern is such that there is no spatial and temporal overlap with the trailing bunches, both considering the time-structure of the electron pulses at CLEAR and CLARA. Future tests will be conducted for radiators with larger apertures, more interesting for usual beam instrumentation, and for which the production of and the interaction with the wakefields is even more negligible. To the best knowledge of the authors, this is the first detailed demonstration of the use of CChDR as noninvasive beam diagnostics. Due to the lack of fundamental limitation for resolution in bunch length measurement with coherent radiation technique, CChDR could be extended to extremely short bunch length facilities, e.g., free electron lasers and plasma or dielectric accelerators, where coherent radiation is emitted in the near infrared or visible range. In this case suitable materials should be selected for avoiding possible dispersion of ChDR propagating within the radiator. The detection system could be then directly based on optical fibers, reaching very small dimensions. Typical peak powers are reaching values around the MW threshold, which provide large and easily detectable output signals, also interesting for $\mathrm{THz}$ generation in already-existing, large-scale central and/or futuristic radiation facilities providing additional research opportunities for the users. This opens the possibility for further optimization reducing the sensor in size and providing, as such, compact non-invasive instruments. Undoubtedly, the use of CChDR for generating high peak power in the $\mathrm{THz}$ frequencies requires additional investigation and optimization, in particular of the radiator design. This, as well as dedicated studies on the use of CChDR for beam position monitoring with a more interesting design in terms of accelerator technology, will be the topic of upcoming papers.

\section{ACKNOWLEDGMENTS}

K. L.'s contribution was supported by the Competitiveness Programme of National Research Nuclear University "MEPhI (Moscow Engineering Physics Institute)."

\section{APPENDIX: FOURIER TRANSFORM OF A SKEW-GAUSSIAN DISTRIBUTION}

In this work we define the skew-Gaussian distribution as:

$$
S k G(t)=e^{-\frac{t^{2}}{2 \sigma_{\tau}^{2}}}[1+\operatorname{erf}(\alpha t)]
$$




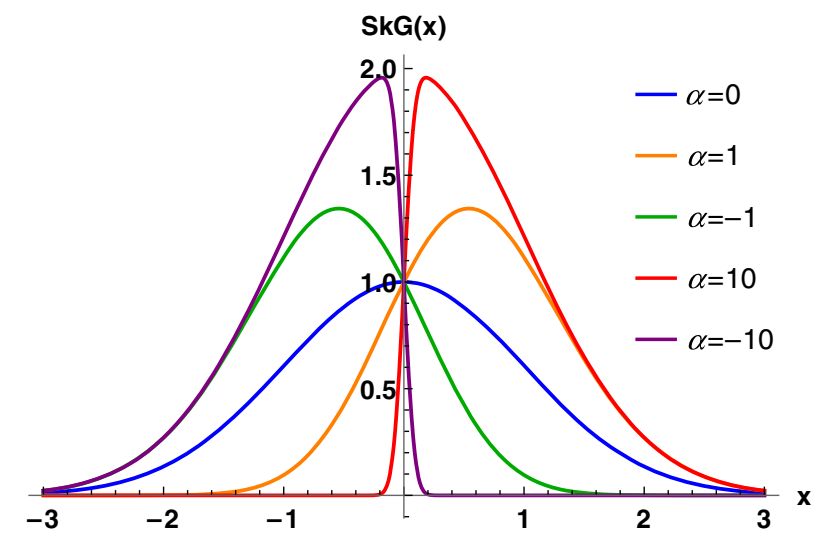

FIG. 13. Skew Gaussian distribution for different $\alpha$ 's.

the parameter $\alpha$ giving a measure of the skewness of the distribution (Fig. 13). It is also worth noting that the sign of $\alpha$ determines if the skewness is along the rising or the falling edge of the distribution. From now on we consider a skew-gaussian electron current density $j(t)$ :

$$
j(t)=j_{0} e^{-\frac{t^{2}}{2 \sigma_{\tau}^{2}}}[1+\operatorname{erf}(\alpha t)]
$$

where $j_{0}$ is the peak current density. We finally recall that the spectrum of coherent radiation $d I / d \omega$ emitted by this electron bunch is $\propto\left|j\left(\omega, \sigma_{\tau}, \alpha\right)\right|^{2}$, where $j\left(\omega, \sigma_{\tau}, \alpha\right)$ is the Fourier transform of the electron current density. In order to get an explicit expression for $j\left(\omega, \sigma_{\tau}, \alpha\right)$, we shall solve the integral:

$$
j\left(\omega, \sigma_{\tau}, \alpha\right)=j_{0} \int_{-\infty}^{\infty} e^{-\frac{t^{2}}{2 \sigma_{\tau}^{2}}}[1+e r f(\alpha t)] e^{-i \omega t} d t .
$$

First of all we solve the trivial part of the integral:

$$
j_{0} \int_{-\infty}^{\infty} e^{-\frac{t^{2}}{2 \sigma_{\tau}^{2}}} e^{-i \omega t} d t=j_{0} e^{-\frac{\sigma_{\tau}^{2} \omega^{2}}{2}}
$$

Then we move to solve the second part, involving the erf function. The first step is to combine the terms in the arguments of the exponential functions in Eq. (A3) in such a way to obtain the following expression:

$$
j_{0} e^{-\frac{\sigma_{\tau}^{2} \omega^{2}}{2}} \int_{-\infty}^{\infty} e^{-\frac{1}{2}\left(\frac{t}{\sigma_{\tau}}+i \sigma_{\tau} \omega\right)^{2}} \operatorname{erf}(\alpha t) d t
$$

Now we derive the integral expression in Eq. (A5) by $\omega$ :

$$
\begin{aligned}
\partial_{\omega} & {\left[\int_{-\infty}^{\infty} e^{-\frac{1}{2}\left(\frac{t}{\sigma_{\tau}}+i \sigma_{\tau} \omega\right)^{2}} \operatorname{erf}(\alpha t) d t\right] } \\
& =-i \sigma_{\tau} \int_{-\infty}^{\infty} e^{-\frac{1}{2}\left(\frac{t}{\sigma_{\tau}}+i \sigma_{\tau} \omega\right)^{2}}\left(\frac{t}{\sigma_{\tau}}+i \sigma_{\tau} \omega\right) \operatorname{erf}(\alpha t) d t
\end{aligned}
$$

The expression (A6) can be then integrated by parts:

$$
\left.i \sigma_{\tau}^{2} e^{-\frac{1}{2}\left(\frac{t}{\tau}+i \sigma_{\tau} \omega\right)^{2}} \operatorname{erf}(\alpha t)\right|_{-\infty} ^{\infty}-\frac{2 i \alpha \sigma_{\tau}^{2}}{\sqrt{\pi}} \int_{-\infty}^{\infty} e^{-\frac{1}{2}\left(\frac{t}{\sigma_{\tau}}+i \sigma_{\tau} \omega\right)^{2}} e^{-\alpha^{2} t^{2}} d t
$$

where the first term has been crossed out because null at $\pm \infty$. The Eq. (A7) can be thus integrated over $t$ :

$$
-\frac{2 i \alpha \sigma_{\tau}^{2}}{\sqrt{\pi}} \int_{-\infty}^{\infty} e^{-\frac{1}{2}\left(\frac{t}{\sigma_{\tau}}+i \sigma_{\tau} \omega\right)^{2}} e^{-\alpha^{2} t^{2}} d t=\frac{2 \sqrt{2} i \alpha \sigma_{\tau}^{3}}{\sqrt{1+2 \alpha^{2} \sigma_{\tau}^{2}}} e^{-\frac{\alpha^{2} \sigma_{\tau}^{4} \omega^{2}}{1+2 \alpha^{2} \sigma_{\tau}^{2}}}
$$

To conclude, we use Eq. (A5) and integrate Eq. (A8) by $\omega$ in order to obtain the final expression for $j\left(\omega, \sigma_{\tau}, \alpha\right)$ :

$$
\begin{aligned}
j\left(\omega, \sigma_{\tau}, \alpha\right) & =j_{0} e^{-\frac{\sigma_{\tau}^{2} \omega^{2}}{2}} \int_{0}^{\omega} \frac{2 \sqrt{2} i \alpha \sigma_{\tau}^{3}}{\sqrt{1+2 \alpha^{2} \sigma_{\tau}^{2}}} e^{-\frac{\alpha^{2} \sigma_{\tau}^{4} \omega^{2}}{1+2 \alpha^{2} \sigma_{\tau}^{2}}} d \omega \\
& =\sqrt{2 \pi} j_{0} \sigma_{\tau} e^{-\frac{\sigma_{\tau}^{2} \omega^{2}}{2}} \operatorname{erf}\left(i \frac{\alpha \sigma_{\tau}^{2} \omega}{\sqrt{1+2 \alpha^{2} \sigma_{\tau}^{2}}}\right) .
\end{aligned}
$$

The bunch form factor is finally obtained as the absolute square of Eq. (A9), i.e., $\left|j\left(\omega, \sigma_{\tau}, \alpha\right)\right|^{2}$.

[1] P. Čerenkov, Visible radiation produced by electrons moving in a medium with velocities exceeding that of light, Phys. Rev. 52, 378 (1937).

[2] I. Tamm, Radiation emitted by uniformly moving electrons, J. Phys. (USSR) 1, 439 (1939).

[3] T. Kajita, Nobel lecture: Discovery of atmospheric neutrino oscillations, Rev. Mod. Phys. 88, 030501 (2016).

[4] M. Demarteau, R. Lipton, H. Nicholson, and I. Shipsey, Particle and nuclear physics instrumentation and its broad connections, Rev. Mod. Phys. 88, 045007 (2016).

[5] C. W. Fabjan and F. Gianotti, Calorimetry for particle physics, Rev. Mod. Phys. 75, 1243 (2003).

[6] R. Kieffer, L. Bartnik, M. Bergamaschi, V. Bleko, M. Billing, L. Bobb, J. Conway, M. Forster, P. Karataev, A. Konkov et al., Direct Observation of Incoherent Cherenkov Diffraction Radiation in the Visible Range, Phys. Rev. Lett. 121, 054802 (2018).

[7] M. Danos, Čerenkov radiation from extended electron beams, J. Appl. Phys. 26, 2 (1955).

[8] Y. Kalkal and V. Kumar, Analysis of čerenkov free-electron lasers, Phys. Rev. ST Accel. Beams 18, 030707 (2015).

[9] J. Walsh, T. Marshall, and S. Schlesinger, Generation of coherent cerenkov radiation with an intense relativistic electron beam, Phys. Fluids 20, 709 (1977).

[10] W. Main, R. Cherry, and E. Garate, $200 \mathrm{mw}$ s-band dielectric cherenkov maser oscillator, Appl. Phys. Lett. 55, 1498 (1989).

[11] W. Peter, E. Garate, W. Main, and A. Fisher, High-gain x-band dielectric cherenkov maser, Phys. Rev. Lett. 65, 2989 (1990). 
[12] J. Hummelt, X. Lu, H. Xu, I. Mastovsky, M. Shapiro, and R. Temkin, Coherent Cherenkov-Cyclotron Radiation Excited by an Electron Beam in a Metamaterial Waveguide, Phys. Rev. Lett. 117, 237701 (2016).

[13] F. Ciocci, A. Doria, G. Gallerano, I. Giabbai, M. Kimmitt, G. Messina, A. Renieri, and J. Walsh, Observation of Coherent Millimeter and Submillimeter Emission from a Microtron-Driven Cherenkov Free-Electron Laser, Phys. Rev. Lett. 66, 699 (1991).

[14] J. Ohkuma, S. Okuda, and K. Tsumori, Measurement of Coherent Cherenkov Radiation from an Intense Beam of a Picosecond Electron Bunch, Phys. Rev. Lett. 66, 1967 (1991).

[15] W. Gai, P. Schoessow, B. Cole, R. Konecny, J. Norem, J. Rosenzweig, and J. Simpson, Experimental Demonstration of Wake-field Effects in Dielectric Structures, Phys. Rev. Lett. 61, 2756 (1988).

[16] B. O'Shea, G. Andonian, S. Barber, K. Fitzmorris, S. Hakimi, J. Harrison, P. Hoang, M. Hogan, B. Naranjo, O. Williams et al., Observation of acceleration and deceleration in gigaelectron-volt-per-metre gradient dielectric wakefield accelerators, Nat. Commun. 7, 12763 (2016).

[17] M. Shevelev, H. Deng, A. Potylitsyn, G. Naumenko, J. Zhang, S. Lu, S. Gogolev, and D. Shkitov, Sub-millimeter bunch length non-invasive diagnostic based on the diffraction and cherenkov radiation, J. Phys. Conf. Ser. 357, 012023 (2012).

[18] A. Tremaine, J. Rosenzweig, S. Anderson, P. Frigola, M. Hogan, A. Murokh, C. Pellegrini, D. Nguyen, and R. Sheffield, Observation of Self-Amplified Spontaneous-Emission-InducedElectron-Beam Microbunching Using Coherent Transition Radiation, Phys. Rev. Lett. 81, 5816 (1998).

[19] A. Lumpkin, R. Dejus, W. Berg, M. Borland, Y. Chae, E. Moog, N. Sereno, and B. Yang, First Observation of z-Dependent Electron-Beam Microbunching Using Coherent Transition Radiation, Phys. Rev. Lett. 86, 79 (2001).

[20] A. Lumpkin, R. Dejus, J. Lewellen, W. Berg, S. Biedron, M. Borland, Y. Chae, M. Erdmann, Z. Huang, K.-J. Kim et al., Evidence for Microbunching "Sidebands" in a Saturated Free-Electron Laser Using Coherent Optical Transition Radiation, Phys. Rev. Lett. 88, 234801 (2002).

[21] Y. Glinec, J. Faure, A. Norlin, A. Pukhov, and V. Malka, Observation of Fine Structures in Laser-Driven Electron Beams Using Coherent Transition Radiation, Phys. Rev. Lett. 98, 194801 (2007).

[22] O. Lundh, C. Rechatin, J. Lim, V. Malka, and J. Faure, Experimental Measurements of Electron-Bunch Trains in a Laser-Plasma Accelerator, Phys. Rev. Lett. 110, 065005 (2013).

[23] M. Veronese, R. Appio, P. Craievich, and G. Penco, Absolute Bunch Length Measurement Using Coherent Diffraction Radiation, Phys. Rev. Lett. 110, 074802 (2013).

[24] Y. Liang, Y. Du, X. Su, D. Wang, L. Yan, Q. Tian, Z. Zhou, D. Wang, W. Huang, W. Gai et al., Observation of coherent smith-purcell and transition radiation driven by single bunch and micro-bunched electron beams, Appl. Phys. Lett. 112, 053501 (2018).

[25] A. Curcio, M. Anania, F. Bisesto, M. Botton, M. Castellano, E. Chiadroni, A. Cianchi, M. Ferrario, M. Galletti, D. Giulietti et al., Electro-optical detection of coherent radiation induced by relativistic electron bunches in the near and far fields, Phys. Rev. Applied 9, 024004 (2018).

[26] D. Karlovets and A. Potylitsyn, Universal description for different types of polarization radiation, arXiv:0908.2336.

[27] D. Smithe and L. Ludeking, An eigenmode solution algorithm based on high-order power iteration with fractally ordered shifts, Comput. Phys. Commun. 106, 95 (1997).

[28] M. V. Shevelev and A. S. Konkov, Peculiarities of the generation of vavilov-cherenkov radiation induced by a charged particle moving past a dielectric target, J. Exper. Theor. Phys. 118, 501 (2014).

[29] C. Nieter and J.R. Cary, Vorpal: a versatile plasma simulation code, J. Comput. Phys. 196, 448 (2004).

[30] A. D. Greenwood, K. L. Cartwright, J. W. Luginsland, and E. A. Baca, On the elimination of numerical cerenkov radiation in pic simulations, J. Comput. Phys. 201, 665 (2004).

[31] P. Karataev, Pre-wave zone effect in transition and diffraction radiation: Problems and solutions, Phys. Lett. A 345, 428 (2005).

[32] K. Sjobak, E. Adli, M. Bergamaschi, S. Burger, R. Corsini, A. Curcio, S. Curt, S. Döbert, W. Farabolini, D. Gamba et al., Status of the clear electron beam user facility at cern, in 10th International Partile Accelerator Conference (IPAC'19), Melbourne, Australia, 19-24 May 2019 (JACOW Publishing, Geneva, Switzerland, 2019), pp. 983-986.

[33] A. Curcio, M. Bergamaschi, R. Corsini, D. Gamba, W. Farabolini, T. Lefevre, S. Mazzoni, V. Dolci, M. Petrarca, and S. Lupi, Beam-based sub-thz source at the cern linac electron accelerator for research facility, Phys. Rev. Accel. Beams 22, 020402 (2019).

[34] L. Garolfi, I. Gorgisyan, H. Purwar, P. Lepercq, D. Gamba, W. Farabolini, S. Döbert, R. Corsini, A. Curcio, C. Vallerand et al., Jacow: Beam dynamics studies and instrumentation tests for bunch length measurements at clear, Proceeding of 29th International Linear Accelerator Conference, Beijing, China, 2018 (2018), pp. MOPO020, https://doi.org/10.18429/JACoW-LINAC2018-MOPO020.

[35] P. McIntosh, D. Scott, S. Jamison, B. Fell, P. Williams, T. Jones, Y. Saveliev, N. Thompson, L. Cowie, B. Militsyn et al., The vela and clara test facilities at daresbury laboratory, Proceeding of 28th International Linear Accelerator Conference (LINAC16) (2017), https://doi.org/ 10.18429/JACoW-LINAC2016-TH3A03.

[36] D. Martin and E. Puplett, Polarised interferometric spectrometry for the millimetre and submillimetre spectrum, Infrared Phys. 10, 105 (1970).

[37] http://terasense.com/products/sub-thz-imaging-cameras, (2019).

[38] R. Bretzlaff and T. Bahder, Apodization effects in fourier transform infrared difference spectra, Revue de physique appliquée 21, 833 (1986).

[39] M. Micheler, R. Ainsworth, G. Blair, G. Boorman, R. Corsini, P. Karataev, T. Lefevre, and K. Lekomtsev, Longitudinal beam profile monitor at $\mathrm{ctf} 3$ based on coherent diffraction radiation, J. Phys. Conf. Ser. 236, 012021 (2010).

[40] R. Lai, U. Happek, and A. Sievers, Measurement of the longitudinal asymmetry of a charged particle bunch from the coherent synchrotron or transition radiation spectrum, Phys. Rev. E 50, R4294 (1994). 